\title{
Obesidad, riesgo cardiovascular y actividad física en estudiantes de Medicina de tres universidades colombianas. Estudio multicéntrico
}

\author{
Obesity, cardiovascular risk, and physical activity in medical students of \\ 3 Colombian universities. Multicenter study
}

\author{
Bados-Enríquez DM ${ }^{1,2}$, Bilbao-Acosta DS ${ }^{1}$, Basante-Gómez JL ${ }^{1}$, Benavides-Castellanos LM ${ }^{1}$, \\ Santofimio-Bernal $\mathrm{OA}^{3}$, Martínez $A^{4}$, Mejía-Gonzales $A M^{5}$.
}

\author{
${ }^{1}$ Facultad de Ciencias de la Salud, Universidad del Tolima; Ibagué, \\ Colombia. \\ ${ }^{2}$ Red Colombiana de Investigación en Diabetes Mellitus (RECI-DM). \\ ${ }^{3}$ Médico interno, Universidad del Tolima. \\ ${ }^{4}$ Facultad de Ciencias de la Salud, Universidad del Norte; \\ Barranquilla, Colombia. \\ ${ }^{5}$ Facultad de Ciencias de la Salud. Universidad CES; Medellín, \\ Colombia.
}

Autor de correspondencia: Diego Mauricio Bados Enríquez Correo electrónico: diegoconques@gmail.com

Fecha de recepción: 26/04/2020

Fecha de aceptación: 31/08/2020

\section{Resumen}

Introducción: un cambio importante en salud pública que tuvo el siglo XXI fue el espectro del aumento del peso corporal como una pandemia. En la población de jóvenes y adultos en Colombia, 1 de cada 3 tiene sobrepeso $(37,7 \%)$, mientras que 1 de cada 5 es obeso (18,7\%).

Metodología: se realizó un estudio multicéntrico descriptivo de corte transversal, en el que participaron tres universidades colombianas. El análisis de los datos se llevó a cabo mediante análisis descriptivo para las variables de interés.

Resultados: se seleccionaron 233 estudiantes de Medicina. Al analizar el índice de masa corporal (IMC), la mayoría de estos se encontraba en un rango de normalidad, con un $75 \%$, seguida por un 19,9\% correspondiente a los que clasificaron como sobrepeso. Con respecto a la actividad física, encontramos que el 46,7 \% de los individuos realizan un alto nivel de actividad física.

Discusión: nuestro estudio es acorde con la realidad global, ya que el 19,9 \% de la población estudiada tiene sobre- peso. En cuanto a la actividad física, el 58,7 \% lleva una vida sedentaria, evidenciada por el tiempo que se permanece sentado; de esta población, 110 individuos permanecen entre 6 y 10 horas sentados.

Conclusiones: la investigación sobre los factores de riesgo cardiovascular, obesidad y metabolismo cada vez se centra más en empezar a analizar estas variables desde sus inicios para evitar en un futuro la enfermedad cardiovascular.

Palabras clave: ejercicio físico, estudiantes, factores de riesgo, obesidad, enfermedades cardiovasculares.

\section{Abstract}

Introduction: An important change in public health that the 21st century had was the spectrum of body weight gain as a pandemic. In Colombia, of young people and adults, one in three is overweight (37.7\%), while one in five is obese (18.7\%).

Methodology: A descriptive multicentre cross-sectional study will be carried out. 3 Colombian universities participated. The data analysis performed a descriptive analysis for the variables of interest.

Results: 233 medical students were selected. When analyzing the body mass index (BMI) the largest number of people is in a normal range with $75 \%$ followed by a $19.9 \%$ who classify as overweight. With respect to physical activity, we will find $46.7 \%$ of the individuals carried out a high level of physical activity.

Discussion: Our study is consistent with the global reality, since $19.9 \%$ are overweight. Physical activity where $58.7 \%$ lead a sedentary life evidenced by the time that they remain seated where 110 people stay between 6 and 10 hours.

Conclusions: Research on cardiovascular risk factors, obesity and metabolism increasingly focuses on beginning to 
analyze these variables from the beginning to avoid cardiovascular disease in the future.

Keywords: Exercise; Student; Risk factors; Obesity; Cardiovascular diseases.

\section{Introducción}

Un cambio importante en salud pública que tuvo el siglo XXI fue el aumento del peso corporal como una pandemia (1). Analizando la Encuesta Nacional de Situación Nutricional de Colombia-2015 (ENSIN) observamos que, en jóvenes y adultos (18-64 años), 1 de cada 3 tiene sobrepeso (37,7 \%), mientras que 1 de cada 5 es obeso (18,7 \%) (2). En este sentido, el 56,4\% de la población presenta exceso de peso, lo que significa un incremento de 5,2 puntos porcentuales con respecto al 2010 (3). Aproximadamente la mitad de los adultos colombianos realiza 150 minutos semanales de actividad física moderada o $75 \mathrm{mi}-$ nutos semanales de actividad vigorosa o fuerte. En promedio, 4 de cada 10 mujeres y 6 de cada 10 hombres atienden esta medida de prevención $(4,5)$. Diversos estudios han evidenciado esta situación en estudiantes universitarios $(6,7)$ como una forma de evaluar datos desde la base de la problemática y convertirlos en la piedra angular para cortar con desenlaces que pongan en riesgo la vida de las personas $(8,9)$. Entendiendo que el eje central son los factores de riesgo cardiovasculares y metabólicos en población infantil y adultos jóvenes que determinan el desarrollo de enfermedades crónicas fatales en el adulto, el grupo investigador tiene como objetivo evaluar la presencia de obesidad, riesgo cardiovascular y actividad física en estudiantes de Medicina de tres universidades de Colombia, con el fin de conocer la realidad local, y si es pertinente, a futuro, diseñar estrategias de promoción de estilos de vida saludables en la comunidad universitaria local y nacional.

\section{Metodología}

Se realizó un estudio multicéntrico descriptivo de corte transversal. Participaron la Universidad del Tolima (pública), la Universidad del Norte en Barranquilla (privada) y la Universidad CES de Medellín (privada). La muestra poblacional quedó conformada por 233 estudiantes de pregrado de Medicina. Se excluyeron aquellos estudiantes que no suministraron datos suficientes para la realización de las pruebas, los menores de 18 años y los que manifestaron no querer ser parte del estudio. El tamaño de la muestra se calculó asumiendo un nivel de confianza del $95 \%$, una heterogeneidad del $50 \%$, que buscaba representar las diferencias de variables y resultados entre géneros, y un margen de error del 5 \%. Luego se realizó un muestreo aleatorio simple por medio de Epilnfo 7. A los estudiantes seleccionados se les invitó a participar en el estudio y aquellos que no accedieron fueron sustituidos por otros. Se debe aclarar que no se realizó la intervención a todos los estudiantes matriculados de las universidades, sino solamente hasta alcanzar el número mencionado de la muestra.

Se realizaron jornadas de intervención en las tres universidades y de manera presencial se aplicó un cuestionario por medio del cual se recolectaron variables como: género, edad, semestre en curso, tabaquismo (por medio de cuestionario de la Organización Mundial de la Salud [OMS]) (10), sedentarismo (evaluado por el Cuestionario Internacional de Actividad Física-IPAQ) (11), antecedentes familiares de hipertensión arterial (HTA), diabetes mellitus (DM) o enfermedades cardiovasculares (ECV). Se dispuso de un espacio para la toma de medidas antropométricas como la talla y el peso por medio de básculas previamente calibradas el día anterior. Se calculó el índice de masa corporal (IMC). Se tomó el índice cinturacadera del perímetro abdominal, evaluado según los valores dados por la OMS, el perímetro abdominal, medido por debajo de las costillas, a nivel del ombligo, y se clasificó en rangos: para hombres, $<94 \mathrm{~cm}$ y $94-102 \mathrm{~cm}$ o $>102$; para mujeres, $<80$ cm y $80-88 \mathrm{~cm},>88 \mathrm{~cm}$, respectivamente. Estos datos se ingresaron en una base de datos en Excel 2017.

El análisis de los datos se llevó a cabo por medio de un análisis descriptivo para las variables de interés. Para las variables cuantitativas se realizaron medidas de tendencia central y de dispersión. En las variables de razón, como edad, peso, talla, IMC y presión arterial, se calcularon la media, la mediana, la desviación estándar y la moda; con estas variables se llevó a cabo una regresión lineal. En las variables nominales, como el género, se analizaron las proporciones. La clasificación y su caracterización se realizó por medio de los valores que proporcionaron los instrumentos.

El trabajo de investigación se realizó amparado por el comité de ética de la Universidad del Tolima, igualmente por la Ley 8430 de 1993 de Colombia, por la cual se establecen las normas científicas, técnicas y administrativas para la investigación en salud y se cataloga esta investigación como sin riesgo. El consentimiento informado se manejó por medio de un formato donde se explicaba a la persona encuestada las acciones que implementamos como investigadores y el uso de los datos obtenidos.

\section{Resultados}

La muestra seleccionada fue de 233 estudiantes de Medicina de tres universidades de Colombia. De esta muestra, la mayoría de los estudiantes es de género femenino (58,7 \%). La edad correspondió a una media de 20,3 años. Estos estudiantes provenían de todos los semestres, excepto del año de internado; la media fue de 5,8 semestres. Con respecto a las medidas de peso, se obtuvo una media de $63,4 \mathrm{~kg}$ y una talla de un valor mínimo de 1,53 cm y un valor máximo de $1,83 \mathrm{~cm}$, con lo cual se calculó el IMC y se obtuvo una media de $22,8 \mathrm{~kg} / \mathrm{m}^{2}$. Con respecto a los antecedentes familiares sobre enfermedad 
cardiovascular, HTA o diabetes, el 65,8 \% respondió afirmativamente y el $34,3 \%$ lo hizo negativamente. Todos estos datos se pueden ver en la Tabla 1.

Tabla 1. Características de la muestra estudiada

\begin{tabular}{|c|c|}
\hline Género & $\begin{array}{l}\text { F: } 137(58,7 \%) \\
\text { M: } 96(41,2 \%)\end{array}$ \\
\hline Edad & 20,3 (DE: 1,9 años; IC 95 \%: 20,07-20,53) \\
\hline Semestre & 5,8 (DE: 1,9 semestres; IC $95 \%: 5,57-6,03$ ) \\
\hline Universidad & $\begin{array}{l}\text { Universidad del Tolima } \\
\text { Universidad del Norte } \\
\text { Universidad CES }\end{array}$ \\
\hline Peso & 63,4 kg (DE: 12,2 kg; IC 95 \%: 61,9-64,8) \\
\hline Talla & $\begin{array}{l}\text { Mínima: } 1,53 \\
\text { Máxima: } 1,83\end{array}$ \\
\hline IMC & 22,8 (DE: 3,2 kg/m²; IC: $22,4-23,1$ ) \\
\hline $\begin{array}{l}\text { Antecedentes de } \\
\text { enfermedades } \\
\text { cardiovasculares, } \\
\text { diabetes o HTA }\end{array}$ & $\begin{array}{l}\text { Sí: } 153 \text { (65,6 \%) } \\
\text { No: } 80 \text { (34,3 \%) }\end{array}$ \\
\hline
\end{tabular}

DE: desviación estándar; F: femenino; HTA: hipertensión arterial alta; IC: intervalo de confianza; IMC: índice de masa corporal; M: masculino.

Al analizar el IMC, como se muestra en la Tabla 2, podemos observar que la mayoría de las personas se encontraba en un rango de normalidad, con un $75 \%$ (n=193), seguida por un $19,9 \%(n=51)$, correspondiente a los que estaban en el rango de sobrepeso. Es alarmante que, en esta población, que en promedio tiene 20 años, encontráramos a un estudiante en el rango de obesidad extrema, con un IMC mayor de $40 \mathrm{~kg} / \mathrm{m}^{2}$.

Tabla 2. Índice de masa corporal

\begin{tabular}{l|c|c|c}
\hline \multicolumn{1}{c|}{ Clasificación } & $\begin{array}{c}\text { Valores de } \\
\text { referencia }\end{array}$ & $\mathbf{n}$ & $\%$ \\
\hline Infrapeso & $<18 \mathrm{~kg} / \mathrm{m}^{2}$ & 3 & 1,17 \\
\hline Normal & $\begin{array}{c}18,5 \mathrm{~kg} / \mathrm{m}^{2} \mathrm{a} \\
24,9 \mathrm{~kg} / \mathrm{m}^{2}\end{array}$ & 193 & 75 \\
\hline Sobrepeso & $\begin{array}{c}25 \mathrm{~kg} / \mathrm{m}^{2} \mathrm{a} \\
29,9 \mathrm{~kg} / \mathrm{m}^{2}\end{array}$ & 51 & 19,9 \\
\hline Obesidad & $\begin{array}{c}30 \mathrm{~kg} / \mathrm{m}^{2} \mathrm{a} \\
34,9 \mathrm{~kg} / \mathrm{m}^{2}\end{array}$ & 4 & 1,5 \\
\hline Obesidad extrema & $\geq 40 \mathrm{~kg} / \mathrm{m}^{2}$ & 1 & 0,3 \\
\hline
\end{tabular}

El índice cintura-cadera es una medida que nos permite determinar, a partir de la acumulación de grasa abdominal, el riesgo cardiovascular. En nuestro estudio encontramos que, con respecto al género femenino, la mayoría de la población tiene un muy bajo riesgo cardiovascular $(72,9 \%$; $=64)$. Si observamos los rangos del género masculino, veremos la misma tendencia al encontrar que la mayoría presenta una medida promedio del índice menor de 0,95 cm (77,08 \%; n=74). Es importante recalcar que incluso en esta población joven encontramos un gran porcentaje de mujeres con riesgo cardiovascular alto $(27,7 \%)$, que sobrepasa en 6 veces el porcentaje de riesgo cardiovascular alto en hombres (4,16\%), como se puede ver en la Tabla 3.

Tabla 3. Índice cintura-cadera y riesgo cardiovascular

\begin{tabular}{c|l|c|c|c}
\hline \multicolumn{1}{c|}{ ICC } & $\begin{array}{l}\text { Valores de } \\
\text { referencia }\end{array}$ & $\begin{array}{c}\text { Riesgo } \\
\text { cardiovascular }\end{array}$ & $\mathbf{n}$ & $\%$ \\
\hline \multirow{2}{*}{ Femenino } & $\begin{array}{l}\text { Menos de } \\
0,80\end{array}$ & Muy bajo & 64 & $72,9 \%$ \\
\cline { 2 - 5 } & 0,81 a 0,84 & Bajo & 35 & $25,5 \%$ \\
\cline { 2 - 5 } & $\begin{array}{l}\text { Igual o más } \\
\text { de 0,84 }\end{array}$ & Alto & 38 & $27,7 \%$ \\
\hline \multirow{2}{*}{ Masculino } & $\begin{array}{l}\text { Menos de } \\
0,95\end{array}$ & Muy bajo & 74 & $77,08 \%$ \\
\cline { 2 - 5 } & 0,96 a 0,99 & Bajo & 18 & $18,75 \%$ \\
\cline { 2 - 5 } & $\begin{array}{l}\text { Igual o más } \\
\text { de 1 }\end{array}$ & Alto & 4 & $4,16 \%$ \\
\hline
\end{tabular}

ICC: índice cintura-cadera.

El análisis de los datos para fumadores se realizó por medio del cuestionario de la OMS, donde encontramos que solo el $4,29 \%(n=10)$ fuma, y de estos, el $100 \%$ se clasifica como fumadores leves, como se puede ver en la Tabla 4.

Con respecto a la actividad física, encontramos que el $46,7 \%$ y el $34,2 \%$ de los individuos realizaban un alto y un moderado nivel de actividad física, respectivamente. Un dato relevante es que si calculamos los MET de las personas que caminaban 7 días a la semana por lo menos durante 1 hora obtendremos una cifra de 1386, lo que los cataloga como un moderado nivel de actividad, aunque solamente caminen. Por último, observamos que el 58,7 \% no alcanzaba la medida de 600 MET, lo cual los clasificaba como nivel bajo de actividad física. En contraste, el 15,4 \% no realizaba ninguna actividad física en la semana, datos que se pueden observar en la Tabla 5, en donde se especifica el cálculo de MET. 
Tabla 4. Caracterización de encuestados: fumadores

\begin{tabular}{l|c|c|c|c|c}
\hline \multirow{4}{*}{$\begin{array}{l}\text { Fumadores } \\
223(95,7 \%)\end{array}$} & $\begin{array}{c}\text { Fuma cigarrillo, } \\
\text { tabaco, pipa } \\
\text { y sustancia } \\
\text { psicoactiva }\end{array}$ & $\begin{array}{c}\text { Fuma alrededor } \\
\text { de } 1 \text { o más } \\
\text { cigarrillos, pero } \\
\text { no diariamente }\end{array}$ & $\begin{array}{c}\text { Consume menos } \\
\text { de } 5 \text { cigarrillos } \\
\text { diarios }\end{array}$ & $\begin{array}{c}\text { Fuma una } \\
\text { media de 6 a 15 } \\
\text { cigarrillos diarios }\end{array}$ & $\begin{array}{c}\text { Fuma más de 16 } \\
\text { cigarrillos al día, } \\
\text { en promedio }\end{array}$ \\
\cline { 2 - 6 } & Sí & Sí & No & No & No \\
\cline { 2 - 6 } & Sí & Sí & No & No & No \\
\cline { 2 - 6 } & Sí & Sí & No & No & No \\
\cline { 2 - 6 } & Sí & Sí & Sí & No & No \\
\cline { 2 - 6 } & Sí & So & Sí & No & No \\
\cline { 2 - 6 } & Sí & Sí & Sí & No & No \\
\cline { 2 - 6 } & Sí & Sí & No & No & No \\
\cline { 2 - 6 } & Sí & No & No & No & No \\
\hline
\end{tabular}

Tabla 5. Actividad física (cuestionario Internacional de Actividad Física-IPAQ)

\begin{tabular}{|c|c|c|c|}
\hline Nivel de actividad & Valores de referencia & n & $\%$ \\
\hline \multirow[t]{2}{*}{ Alto } & $\begin{array}{l}\text { Actividad física vigorosa por lo menos } 3 \text { días por semana, logrando un total de al } \\
\text { menos } 1500 \mathrm{MET}^{*}\end{array}$ & 79 & 33,9 \\
\hline & $\begin{array}{l}7 \text { días de cualquier combinación de caminata, con actividad física moderada y/o } \\
\text { actividad física vigorosa, logrando un total de al menos } 3000 \text { MET }\end{array}$ & 30 & 12,8 \\
\hline \multirow[t]{3}{*}{ Moderado } & 3 o más días de actividad física vigorosa por lo menos 20 minutos por día & 58 & 24,8 \\
\hline & 5 o más días de actividad física moderada y/o caminata al menos 30 minutos por día & 22 & 9,44 \\
\hline & $\begin{array}{l}5 \text { o más días de cualquiera de las combinaciones de caminata, actividad física } \\
\text { moderada o vigorosa, logrando como mínimo un total de } 600 \mathrm{MET}\end{array}$ & $125^{* *}$ & 53,6 \\
\hline Bajo & Menores de 600 MET & $137^{* * *}$ & 58,7 \\
\hline Inactivo & Nada de actividad física & 36 & 15,4 \\
\hline
\end{tabular}

*Medida específica del instrumento de recolección de datos (MET/min/semana).

**Si una persona camina 7 días a la semana por lo menos 1 hora obtiene 1386 MET, que equivalen a una actividad física moderada.

***Si una persona camina 30 minutos por 3 días a la semana obtiene 297 MET, cuyo valor no alcanza los 600 MET que se necesitan para que se convierta en una actividad física moderada.

Cuando analizamos el último ítem del cuestionario, podemos observar que la mayoría de las personas (n=110; 47,2\%) pasa entre 6 y 10 horas sentada. Igualmente encontramos que una población considerable $(n=28 ; 12 \%)$ pasa más de 10 horas sentada, como se muestra en la Tabla 6.

\section{Discusión}

El análisis epidemiológico más grande hasta la fecha registrado fue dirigido por el Imperial College de Londres y la OMS, donde se analizaron el peso y la talla de cerca de 130 millones de individuos mayores de 5 años (31,5 millones de 
Tabla 6. Tiempo de estar sentado en un día de trabajo

\begin{tabular}{l|c|c}
\multicolumn{1}{c|}{ Tiempo sentado } & $\mathbf{n}$ & $\%$ \\
\hline 1 hora a 5 horas & 83 & 35,6 \\
\hline 6 horas a 10 horas & 110 & 47,2 \\
\hline Más de 10 horas & 28 & 12 \\
\hline No sabe/no está seguro & 12 & 5,1 \\
\hline
\end{tabular}

edades comprendidas entre los 5 y los 19 años y 97,4 millones de individuos de 20 años o más), el cual mostró que las tasas mundiales de obesidad de la población infantil y adolescente aumentaron desde menos de un $1 \%$ en 1975 hasta casi un 6 $\%$ en 2016. Estas cifras muestran que, conjuntamente, el número de individuos obesos de 5 a 19 años se multiplicó por 10 a nivel mundial (12), pasando de los 11 millones en 1975 a 124 millones en 2016. Además, 213 millones presentaban sobrepeso en 2016, si bien no llegaban al umbral de la obesidad (13). Nuestro estudio se desliza por esa vertiente, ya que el 19,9\% está catalogado como sobrepeso según su IMC y que a estas edades tan bajas (18 años) encontramos una persona que tiene obesidad extrema $(14,15)$. Se habla mucho sobre la condición de obesos metabólicamente sanos como un punto estratégico para intervenir y reducir las enfermedades futuras. Nuestra población se encuentra entre edades de adultos jóvenes que nos permitirían analizar estos desenlaces (16, 17). Otra variable importante es la actividad física, donde el 58,7 \% lleva una vida sedentaria que está relacionada con las cargas académicas grandes que conlleva el pregrado de Medicina, evidenciado por el tiempo que permanecen sentados, con un número de 110 personas que permanecen entre 6 y 10 horas sentadas al día (18). Los factores de riesgo cardiovascular están relacionados con aumento de peso, sedentarismo $y$ agentes externos que aportan al deterioro futuro $(19,20)$; en este caso, observamos que el 4,29 \% son catalogados como fumadores leves.

\section{Conclusiones}

La investigación sobre factores de riesgo cardiovascular, obesidad y metabolismo cada vez se centra más en empezar a analizar estas variables, desde la gestante con diabetes mellitus gestacional, el recién nacido macrosómico, los niños con sobrepeso y obesidad, hasta el último eslabón antes de entrar a la meseta problema, que son los adultos jóvenes. Claramente es necesario continuar dirimiendo sobre este tema y trazar el futuro que nos lleve al control de esta pandemia global.

\section{Conflicto de interés}

Los autores manifiestan no tener conflicto de interés.

\section{Fuente de financiación}

Autofinanciado.

\section{Congresos}

Esta investigación fue presentada en el Congreso Estudiantil Colombiano de Investigación Médica (CECIM), Villa de Leyva, Boyacá, 2017, y en el Congreso Científico Internacional (CCI), Asunción, Paraguay, 2017. En ambos congresos obtuvo el segundo lugar en la categoría de Proyectos Multicéntricos. También se presentó este año en el Congreso Internacional de Endocrinología en Buenos Aires, Argentina, en la categoría de Pósteres de investigación.

\section{Referencias}

1. Al-Thani M, Al-Thani A, Alyafei S, Al-Chetachi W, Khalifa SE, Ahmed A, et al. The prevalence and characteristics of overweight and obesity among students in Qatar. Public Health. 2018;160:143-9. doi: 10.1016/j. puhe.2018.03.020

2. González-Zapata L, Carreño-Aguirre C, Estrada A, Monsalve-Alvarez J, Alvarez LS. Exceso de peso corporal en estudiantes universitarios según variables sociodemográficos y estilo de vida. Rev Chil Nutr. 2017;44(3). doi: 10.4067/S0717-75182017000300251

3. Encuesta nacional de la situación nutricional en Colombia 2010 [Internet]. Ministerio de Salud y Protección Social; 2010. [citado en mayo 12 de 2018]. Disponible en: https://bit.ly/2KwUeqM

4. Hartson KR, Gance-Cleveland B, Amura CR, Schmiege S. Correlates of Physical Activity and Sedentary Behaviors Among Overweight Hispanic School-aged Children. J Pediatr Nurs. 2018;40:1-6. doi: 10.1016/j. pedn.2018.01.019

5. Niu Y, Zhou D, Ma Z. Effect of aerobic exercises on students' physical health indicators. Sci Sports. 2018;33(2):e85-9. doi: 10.1016/j.scispo.2018.01.003
6. Peralta C, Loayza K, Medina-Palomino F, Rojas-Vilca J. Monitoreo domiciliario de presión arterial y factores de riesgo cardiovascular en jóvenes estudiantes de medicina de una universidad privada en Lima, Perú. Rev Med Hered. 2017;28(28):157-165. Disponible en: https://bit.ly/385qER7

7. Morales G, Guillen-Grima F, Muñoz S, Belmar C, Schifferli I, Muñoz A, et al Factores de riesgo cardiovascular en universitarios de primer y tercer año. Rev Med Chile. 2017;145:299-308. Disponible en: https://bit.ly/387Vskk

8. Morales G, Castillo B, Muñoz T, Belmar S, Soto C, Schifferli A, et al. Asociación entre factores de riesgo cardiometabólicos, actividad física y sedentarismo en universitarios chilenos. Nutr Hosp Nutr Hosp Hosp. 2017;34(6):1265-516. doi: 10.20960/nh.1060

9. Chávez-Valenzuela ME, Salazar CM, Hoyos-Ruiz G, Bautista-Jacobo A González-Lomeli D, Ogarrio-Perkins CE. Actividad física y enfermedades crónicas no transmisibles de estudiantes mexicanos en función del género. Retos Nuevas Tendencias En Educación Física, Deporte y Recreación. 2018;33:169-74. Disponible en: https://bit.ly/2JX871x

10. Pérez C, Rodríguez I, Gantiva C. Cuestionario para la clasificación de consumidores de cigarrillo (C4) para jóvenes. Diversitas. 2011;7(2):281-91. Disponible en: https://bit.ly/37lwHBV 
11. Mantilla-Toloza SC, Gómez-Conesa A. El Cuestionario Internacional de Actividad Física. Un instrumento adecuado en el seguimiento de la actividad física poblacional. Rev Iberoam Fisioter Kinesiol. 2007;10(1):48-52. Disponible en: https://bit.ly/3adWf5Z

12. Farhadnejad H, Asghari G, Mirmiran P, Azizi F. Dietary approach to stop hypertension diet and cardiovascular risk factors among 10- to 18-year-old individuals. Pediatr Obes. 2018;13(4):185-94. doi: 10.1111/ijpo.12268

13. NCD Risk Factor Collaboration (NCD-RisC). Worldwide trends in body-mass index, underweight, overweight, and obesity from 1975 to 2016: a pooled analysis of 2416 population-based measurement studies in 128.9 million children, adolescents, and adults. Lancet. 2017;390(10113):2627.42. doi: 10.1016/S0140-6736(17)32129-3

14. Palma L, Mendez C, Arana D, Ortiz J. Identificación del Componente Antropométrico de Estudiantes Pertenecientes a Medicina y Educación Física (Uceva Tuluá). Rev Edu Fis. 2017;35(1). Disponible en: https://bit. ly $/ 34 \mathrm{drfPq}$

15. Damiri B, Aghbar A, Alkhdour S, Arafat Y. Characterization and prevalence of metabolic syndrome among overweight and obese young Palestinian students at An-Najah National University. Diabetes Metab Syndr Clin Res Rev. 2018;12(3):343-8. doi: 10.1016/j.dsx.2017.12.021

16. Pajuelo J, Arbañil H, Gonzales JS, Gamarra D, Torres H, Pando R, et al. Obeso metabólicamente normal. An Fac Med. 2014;75(2):113-8. doi: 10.15381/ anales.v75i2.8386

17. Griera-Borrás JL, Contreras-Gilbert J. ¿Existe el obeso sano? Endocrinol Nutr. 2014;61(1):47-51. doi: 10.1016/j.endonu.2013.03.010

18. Hunter J, Rawlings-Anderson K, Lindsay T, Bowden T, Aitken LM. Exploring student nurses' attitudes towards those who are obese and whether these attitudes change following a simulated activity. Nurse Educ Today. 2018;65:225-31. doi: 10.1016/j.nedt.2018.03.013

19. Kim JY, Yang Y, Sim YJ. Effects of smoking and aerobic exercise on male college students' metabolic syndrome risk factors. J Phys Ther Sci. 2018;30(4):595-600. doi: 10.1589/jpts.30.595

20. Bell CN, Thorpe RJ, Bowie JV, LaVeist TA. Race disparities in cardiovascular disease risk factors within socioeconomic status strata. Ann Epidemiol 2018;28(3):147-52. doi: 10.1016/j.annepidem.2017.12.007 


\section{(0) Hay órganos que sufren y no se ven ${ }^{1}$ La diabetes es más que glucosa ${ }^{1}$}

\section{Uso Temprano}
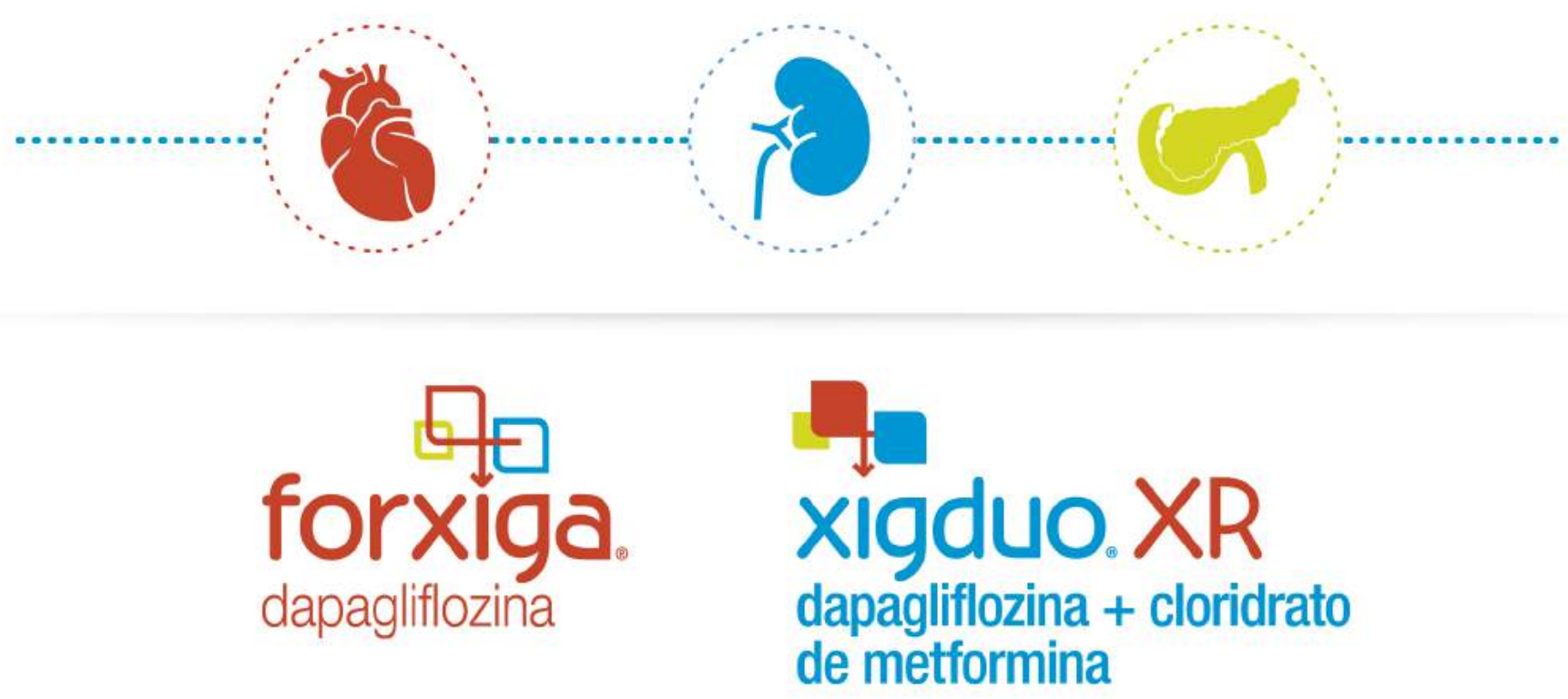

\section{EFICACIA: HbA1c, Peso y PA².8}

\section{AMPLIO PERFIL DE SEGURIDAD2.3}

\section{CONCIENCIA CARDIORRENAL23,9}

Esto puede ocurrir de forma temprana, incluso antes de que su paciente sea dignosticado ${ }^{10}$ ¿Está su paciente con DMT2 protegido?

HbA1c: hemoglobina glucosilada; PA: Presión Arterial; DMT2: diabetes mellitus tipo 2.

Referencias: 1. Chen-Ku CH, et al. Vascular complications in patients with type 2 diabetes: prevalence and comorbidities in 6 countries of Latin America (a cohort of the DISCOVER Study Program). Endocr Pract, 2019 Oct;25(10):994-1002. 2. IPP Forxiga, 3. IPP Xigduo XR. 4. Bailey, et al, Effect of dapagliflozin in patients with 5. Ferrannini E, et al. Diabetes Care 2010;33:2217-24. 6. Bolinder J. Diabetes Obes Metab. 2014 Feb;16(2):159-69. 7. Rosenstock, et al. Composite Endpoint Analysis of Dapaglifiozin versus Saxagliptin as Add-On Therapy in Patients with Type 2 Diabetes inadequately Controlled with Metformin. Presented at the 14th Annual World Congress on Insulin Resistance, Diabetes \& Cardiovascular Disease (WCIRDC), Los Angeles, CA, USA; Supported by: December 1-3, 2016. Rosenstock et al. Arch Endocrinol Metab 2018; 62(4):424-430. 9. Sonesson, et al. Cardiovascular effects of dapagliflozin in patients with type 2 diabetes and

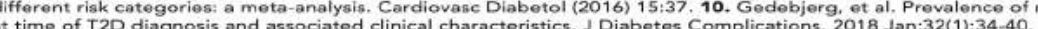

